\title{
Evaluación positiva de medicamentos: octubre 2020
}

\author{
Aparicio-Hernández R. ${ }^{1}$, García-Luque A. ${ }^{2}$, Acuña-Vega A. ${ }^{3}$, Granda-Lobato P. ${ }^{4}$ \\ Sanid. mil. 2021; 77 (1): 29-34, ISSN: 1887-8571
}

\section{RESUMEN}

Se reseñan los medicamentos evaluados y con dictamen positivo por comisión de expertos de la Agencia Española de Medicamentos y Productos Sanitarios o de la Agencia Europea del Medicamento hechos públicos en octubre de 2020 y considerados de mayor interés para el profesional sanitario. Se trata de opiniones técnicas positivas que son previas a la autorización y puesta en el mercado del medicamento.

PALABRAS CLAVE: Cabotegravir (Vocabria ${ }^{\circledR}$ ); Células CD3+ autólogas transducidas anti CD-19 (Tecartus ${ }^{\circledR}$ ); Cultivo celular autólogo de $\mathrm{CD} 34+$, enriquecido con células madre y células progenitoras hematopoyéticas transducidas ex vivo usando un vector lentiviral (lentivirus) con el gen humano arilsulfatasa A (Libmeldy ${ }^{\mathbb{R}}$ ); Fenfluramina (Fintepla ${ }^{\mathbb{R}}$ ); Formoterol $5 \mu \mathrm{G} / 9 \mu \mathrm{G}$ Bromuro de

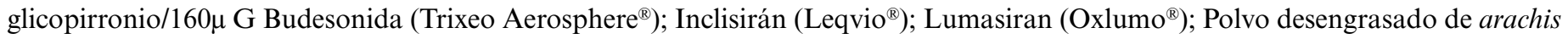
hypogaea 1. (semilla de cacahuete) (Palforzia $\left.{ }^{\circledR}\right)$; Rilpivirina (Rekambys ${ }^{\circledR}$ ).

\section{Positive assessment of drugs: October 2020}

SUMMARY: The drugs assessed by the Spanish Agency for Medicines and Health Products or European Medicines Agency made public in October of 2019, and considered of interest to the healthcare professional, are reviewed. These are positive technical reports prior to the authorization and placing on the market of the product.

KEYWORDS: Cabotegravir (Vocabria ${ }^{\circledR}$ ); Células CD3+ autólogas transducidas anti CD-19 (Tecartus ${ }^{\circledR}$ ); Cultivo celular autólogo de CD34+, enriquecido con células madre y células progenitoras hematopoyéticas transducidas ex vivo usando un vector lentiviral (lentivirus) con el gen humano arilsulfatasa A (Libmeldy ${ }^{\circledR}$ ); Fenfluramina (Fintepla ${ }^{\circledR}$ ); Formoterol $5 \mu$ G/9 $\mu$ G Bromuro de glicopirronio/160 $\mu \mathrm{G}$ Budesonida (Trixeo Aerosphere $\left.{ }^{\circledR}\right)$; Inclisirán $\left(\right.$ Leqvio $\left.^{\circledR}\right)$; Lumasiran $\left(\right.$ Oxlumo $\left.{ }^{\circledR}\right)$; Polvo desengrasado de arachis hypogaea 1. (semilla de cacahuete) $\left(\right.$ Palforzia $\left.^{\circledR}\right)$; Rilpivirina $\left(\right.$ Rekambys $\left.^{\circledR}\right)$.

\section{CABOTEGRAVIR $\left(\text { Vocabria }^{\circledR}\right)^{1-6}$}

Se trata de un antiviral de acción directa, actúa como inhibidor de la transferencia de la cadena de integrasa (INI) mediante su unión al sitio activo de la integrasa del virus de la inmunodeficiencia humana (VIH). Bloquea el proceso de integración del ADN retroviral en el ADN del huésped, impidiendo la replicación del VIH y por tanto manteniendo niveles muy bajos en sangre. No cura la infección por VIH ni el SIDA, pero sí puede frenar el deterioro del sistema inmunitario y la aparición de infecciones y enfermedades asociadas al SIDA.

La indicación aprobada es, en combinación con rilpivirina, para el tratamiento de la infección por el virus de inmunodeficiencia humano tipo 1 (VIH-1) en adultos con carga viral indetectable (HIV-1 RNA < 50 copias $/ \mathrm{mL}$ ) que siguen un régimen antirretroviral estable sin evidencia, ya sea previa o actual, de resistencia o fracaso virológico con inhibidores de la

\footnotetext{
${ }^{1}$ Médico Adjunto. Servicio de Farmacología Clínica.

2 Teniente coronel Médico. Servicio de Farmacología Clínica.

${ }^{3}$ Farmacéutico Adjunto. Servicio de Farmacia Hospitalaria.

${ }^{4}$ Teniente Farmacéutico. Residente segundo año Servicio de Farmacia Hospitalaria.

Hospital Central de la Defensa Gómez Ulla. Madrid. España.
}

Dirección para correspondencia: Servicio de Farmacología Clínica. Hospital Central de la Defensa Gómez Ulla. 28047 Madrid. España. Correo electrónico: Aparicio Hernández, RM. rapaher@mde.es; Acuña Vega, A. aacuveg@mde.es

Recibido: 9 de marzo de 2021

Aceptado:11 de marzo de 2021

doi: $10.4321 /$ S1887-85712021000100005 transcriptasa inversa no análogos de los nucleósidos (ITINN) e inhibidores de la integrasa (INI).

En cuanto a los datos de eficacia y seguridad, proceden principalmente de 2 ensayos clínicos de fase III (FLAIR y ATLAS), aleatorizados, abiertos y controlados llevados a cabo en sujetos infectados por VIH-1 que no habían sido tratados antes con medicamentos para el VIH o que habían tomado estos medicamentos durante al menos seis meses.

Cabotegravir, administrado junto con rilpivirina, resultó ser tan eficaz como otros medicamentos para el VIH a la hora de mantener los niveles de VIH-1 en sangre (viremia) por debajo de un determinado nivel (inferior a 50 copias/ml de ARN del VIH-1).Los pacientes fueron tratados con cabotegravir y rilpivirina o con combinaciones de otros medicamentos, transcurridas 48 semanas, los niveles de VIH-1 estaban por encima del límite en el 1,9\% de los pacientes (11 de 591) que recibían inyecciones de cabotegravir y rilpivirina mensualmente y en el $1,7 \%$ de los pacientes (10 de 591) que recibían otros medicamentos. Un ensayo clínico fase III adicional (ATLAS-2M) mostró que las inyecciones de cabotegravir y rilpivirina administradas mensualmente o cada dos meses eran igualmente eficaces. Transcurridas 48 semanas, para los pacientes que recibían inyecciones cada dos meses, los niveles de VIH-1 estaban por encima del límite en el 1,7\% de los pacientes (9 de 522), en comparación con el 1\% de los pacientes ( 5 de 523 ) que recibían inyecciones mensualmente.

Los efectos adversos más frecuentes que se observaron durante los ensayos clínicos para este régimen en combinación, 
fueron reacciones locales en la zona de la inyección, cefalea, fiebre, náusea, fatiga, astenia, mialgias y mareo.

Debe ser prescrito por médicos con experiencia en el manejo de la infección por VIH y en combinación con rilpivirina. Se tomarán dos comprimidos diarios de cabotegravir y rilpivirina por vía oral durante un mes, tras lo cual se administrarán las inyecciones de ambos, mensualmente o cada dos meses, lo que supone una ventaja. Cabotegravir estará disponible en comprimidos recubiertos con película (30 mg), y en suspensión inyectable de liberación prolongada (400 y $600 \mathrm{mg}$ ).

\section{CÉLULAS CD3+ AUTÓLOGAS TRANSDUCIDAS ANTI - CD19 $\left(\text { Tecartus }^{\circledR}\right)^{1,7-9}$}

La indicación aprobada para Tecartus ${ }^{\circledR}$ es en el tratamiento de pacientes adultos con linfoma de células del manto (LCM) refractario o recurrente, después de dos o más líneas de tratamiento sistémico que incluyan un inhibidor de la tirosina quinasa de Bruton (BTKi).

El linfoma de células del manto se considera raro, y Tecartus ${ }^{\circledR}$ fue designado medicamento huérfano el 13 de noviembre de 2019, lo que implica que la EMA deberá revisar la información disponible hasta el momento para valorar si puede seguir manteniendo la designación de huérfano.

El CHMP ha concedido la autorización condicional, concedida a medicamentos que satisfacen una necesidad médica no cubierta cuando el beneficio de su disponibilidad inmediata supera el riesgo de disponer de menos datos de los que habitualmente se requieren en el momento de la autorización, y por tanto de interés para la salud pública. El titular de la autorización de comercialización se compromete a aportar los datos clínicos completos antes de finalizar 2022 lo que es importante tener en cuenta por parte de los médicos prescriptores.

El principio activo son células CD3+ autólogas anti-CD19 transducidas y modificadas genéticamente ex vivo utilizando un vector retroviral que codifica un receptor de antígeno quimérico anti-CD19 (CAR, por sus siglas en inglés) que comprende un fragmento variable de cadena única $(\mathrm{scFv})$ anti-CD19 murino unido al dominio coestimulador CD28 y al dominio de señalización CD3-zeta. Al unirse a las células tumorales que expresan CD19 y a las células B normales, el medicamento inicia la activación de las células $\mathrm{T}$ y la secreción de citoquinas y quimioquinas inflamatorias. Esta cascada de eventos conduce a la muerte de las células que expresan CD19 presentes en el linfoma del manto.

La eficacia y seguridad de Tecartus ${ }^{\circledR}$ se evaluó en un estudio fase II multicéntrico, abierto y de un solo brazo en pacientes adultos con LCM refractario o en recaída, que habían recibido previamente quimioterapia que contenía antraciclina o bendamustina, un anticuerpo anti-CD20 y BTKi (ibrutinib o acalabrutinib). En total, fueron incluidos y sometidos a la leucaféresis 74 pacientes (definido como ITT), de los que 68 pacientes recibieron tratamiento con Tecartus ${ }^{\circledR}$ en perfusión intravenosa única, con una dosis objetivo de $2 \times 106$ células T CAR anti-CD19/kg (dosis máxima permitida: $2 \times 108$ células) después de la administración de una pauta de tratamiento quimioterápico de linfodepleción que consistía en $500 \mathrm{mg} / \mathrm{m}^{2}$ de ciclofosfamida por vía intravenosa y $30 \mathrm{mg} / \mathrm{m}^{2}$ de fludarabina por vía intravenosa, ambos administrados en el quinto, cuarto y tercer día previos al tratamiento.

La variable principal de eficacia fue la tasa de respuesta objetiva (TRO) determinada por un comité de revisión independiente de acuerdo con los criterios de Lugano de 2014.

Se obtuvo de forma global una la TRO fue del $84 \%$ con (IC95\%: 73,4 a 91,3) y con una tasa de remisión completa (RC) del 59\% (IC95\%: 47,4 a 70,7).

Se definió a priori un conjunto de análisis que se componía de los primeros 60 pacientes tratados con Tecartus ${ }^{\circledR}$, cuya respuesta se evaluó 6 meses después de la evaluación de la enfermedad en la semana 4 después de la perfusión de Tecartus ${ }^{\circledR}$. En este conjunto de análisis de 60 pacientes, la TRO fue del 93\% con una tasa de RC del 67\%. La TRO fue significativamente más alta que la tasa de control histórica preespecificada del $25 \%$ a un nivel de significancia unilateral de $0,025(\mathrm{p}<0,0001)$. Las variables secundarias incluían la duración de la respuesta (DR), la supervivencia global (SG), la supervivencia libre de progresión (SLP) y la gravedad de los acontecimientos adversos.

En relación con la seguridad, se observó de manera muy frecuente síndrome de liberación de citoquinas grave, que puede ser potencialmente mortal, con una mediana del tiempo hasta la aparición de 3 días (intervalo: de 1 a 13 días). Se debe monitorizar estrechamente a los pacientes para detectar signos y síntomas de estos acontecimientos, como fiebre alta, hipotensión, hipoxia, escalofríos, taquicardia y cefalea. Antes de la perfusión de Tecartus ${ }^{\circledR}$ debe estar disponible en el centro al menos 1 dosis de tocilizumab. Otros efectos adversos muy frecuentes fueron infecciones y encefalopatía.

Tecartus ${ }^{\circledR}$ estará disponible como dispersión para perfusión (0,4-2 x $10^{8}$ células) y debe ser prescrito por médicos con experiencia en el tratamiento de tumores hematológicos y formados específicamente para su utilización.

\section{CULTIVO CELULAR AUTÓLOGO DE CD34+, ENRIQUECIDO CON CÉLULAS MADRE Y CÉLULAS PROGENITORAS HEMATOPOYÉTICAS TRANSDUCIDAS EX VIVO CON UN VECTOR LENTIVÍRICO QUE CODIFICA EL GEN HUMANO DE LA ARILSULFATASA A $\left(\text { Libmeldy }^{\circledR}\right)^{1,10-13}$}

Se ha autorizado Libmeldy ${ }^{\circledR}$ en la indicación de tratamiento de la leucodistrofia metacromática (LDM), patología neurodegenerativa rara, de herencia autosómica recesiva caracterizada por una mutación bialélica en el gen de la arilsulfatasa A (ARSA) que se traduce en una reducción en la actividad de la enzima ARSA, una enzima lisosomal relacionada con el metabolismo de los sulfatos, particularmente abundante en las vainas de mielina. Está indicado en niños con 1) LDM infantil tardía o juvenil sin manifestaciones clínicas de la enfermedad»; 2) LDM juvenil con manifestaciones clínicas tempranas, que todavía pueden caminar de forma independiente y en los que aún no ha empezado el deterioro cognitivo.

Es un tipo de medicamento de terapia avanzada (terapia génica), el principio activo son las propias células madre del paciente que han sido modificadas genéticamente para que contengan copias activas del gen ARSA, evitando el acúmulo de 
sulfátidos en el sistema nervioso, así como en otros órganos. Se administra una única vez y por vía intravenosa (en un centro de tratamiento cualificado, con experiencia en trasplantes de células madre hematopoyéticas).

La eficacia y seguridad, se estudió en un ensayo clínico abierto, no aleatorizado, de un solo brazo de tratamiento Libmeldy ${ }^{\circledR}$, en el que se incluyeron 20 con LDM de inicio temprano, con una mediana de seguimiento de 4.0 años (rango: 0,6 a 7,5 años). Las variables co-principales, con la que se midió la eficacia clínica fue 1) la puntuación total obtenida en la escala GMFM (que mide cambios en la función motora gruesa) dos años después de recibir tratamiento, considerando como eficacia un retraso en la progresión del 10\% del total de la puntuación obtenida en la escala por los pacientes tratados comparados con un grupo control histórico; y 2) la actividad de la enzima ARSA medido como un aumento significativo de la actividad ARSA residual en comparación con los valores previos al tratamiento de los sujetos en el año 2 después del tratamiento.

Ambas co-variables se midieron en varios tiempos del seguimiento, de tal manera que los resultados para $n=9$ con un seguimiento de 5,4 años, logró alcanzar el 72,5\% para el objetivo de la escala de GMFM y para la variable actividad ARSA se obtuvo una media de 0,852 nmol/mg/hr (IC95\%: 0,581; 1,25). En n $=11$ con seguimiento de 3,5 años, se obtuvo un $76,5 \%$ y una media de $0,64 \mathrm{nmol} / \mathrm{mg} / \mathrm{hr}$ (IC95\%: 0,37; 1,13), respectivamente.El efecto adverso más frecuente observado fue el desarrollo de anticuerpos frente a ARSA, que parece no afectar a la eficacia. Muchos de los efectos adversos hallados estaban relacionados con el tratamiento de acondicionamiento con busulfán.

La LDM es una enfermedad rara, para la que Libmeldy ${ }^{\circledR}$ fue designado medicamento huérfano el 13 de abril de 2007. La información disponible hasta la fecha deberá ser revisada por la EMA con objeto de determinar si el medicamento puede mantener la designación de huérfano.

\section{FENFLURAMINA (Fintepla $\left.{ }^{\circledR}\right)^{1,14-19}$}

El síndrome de Dravet conocido también como epilepsia mioclónica severa de la infancia, es una forma rara y catastrófica de epilepsia intratable (farmacorresistente) que comienza en el primer año de vida, con una incidencia estimada de 1 cada 16.000 nacimientos.

Fenfluramina es un agente liberador de serotonina y actúa estimulando múltiples subtipos de receptores 5-HT. Puede reducir las convulsiones al actuar como agonista en receptores de serotonina específicos (5-HT1D, 5-HT2A y 5-HT2C), y también al actuar sobre el receptor sigma-1 a nivel central. Aunque realmente se desconoce el mecanismo de acción preciso de la fenfluramina en el síndrome de Dravet.

Se ha autorizado su indicación en el tratamiento de las convulsiones asociadas al Síndrome de Dravet (en combinación con otros antiepilépticos), en pacientes a partir de 2 años. Estará disponible como solución oral $(2,2 \mathrm{mg} / \mathrm{ml})$. El tratamiento debe ser iniciado y supervisado por médicos con experiencia en el tratamiento de la epilepsia. Además, tanto la prescripción como la dispensación deben estar sujetas a un programa de control de acceso al medicamento.
La eficacia, seguridad y tolerabilidad de fenfluramina en niños y adultos jóvenes con síndrome de Dravet se evaluó a través de 2 ensayos clínicos aleatorizados, doble ciego, multicéntricos y controlados con placebo.

El estudio 1 incluyó $n=119$, se establecieron grupos paralelos de 3 brazos de tratamiento y aleatorización 1:1:1 [a una de dos dosis de fenfluramina $(0,7 \mathrm{mg} / \mathrm{kg} /$ día o $0,2 \mathrm{mg} / \mathrm{kg} /$ día, máximo $26 \mathrm{mg} /$ día) o placebo], y con un periodo de tratamiento de 14 semanas. La mediana de la frecuencia basal de las crisis convulsivas cada 28 días fue 34,0, 17,5 y 21,2 en los grupos de placebo, fenfluramina $0,2 \mathrm{mg} / \mathrm{kg} /$ día y fenfluramina $0,7 \mathrm{mg} / \mathrm{kg} /$ día, respectivamente.

El estudio 2 incluyó a $n=87$ ), con grupos paralelos de 2 brazos de tratamiento y aleatorización 1:1 a fenfluramina $0,4 \mathrm{mg} / \mathrm{kg} /$ día (máximo $17 \mathrm{mg} /$ día) o placebo añadido a su régimen de tratamiento estándar estable de estiripentol (más clobazam y/o valproato) y posiblemente otros medicamentos antiepilépticos (controlados de manera inadecuada). Período total de 15 semanas de tratamiento. La mediana de la frecuencia basal de las crisis convulsivas a los 28 días fue de 10,7 y 14,3 en los grupos de placebo y fenfluramina $0,4 \mathrm{mg} / \mathrm{kg} /$ día, respectivamente.

Los efectos adversos más frecuentes fueron falta de apetito, diarrea, fiebre, fatiga, infecciones de vías respiratorias altas, letargia, somnolencia y bronquitis.

Aunque el conocido riesgo de valvulopatía cardiaca e hipertensión arterial pulmonar está asociado a dosis de fenfluramina superiores a las normalmente utilizadas para el síndrome de Dravet, es obligatorio establecer un programa de monitorización (ecocardiograma).

Fintepla $^{\circledR}$ fue designado medicamento huérfano el 16 de enero de 2014.

\section{FORMOTEROL $5 \mu \mathrm{G} / 9 \mu \mathrm{G}$ BROMURO DE GLICOPIRRONIO/160 $\boldsymbol{\mu G}$ BUDESONIDA \\ (Trixeo Aerosphere $\left.^{\circledR}\right)^{1,20-23}$}

Formoterol (agonista del receptor beta2 adrenérgico de larga duración) / bromuro de glicopirronio (antagonista muscarínico de larga duración) / budesonida (corticoide) es una combinación de principios activos inhalados, que actúan tanto sobre el control de los síntomas como en la prevención de las exacerbaciones de la enfermedad pulmonar obstructiva crónica.

El formoterol y el bromuro de glicopirronio inducen la relajación del músculo liso bronquial y producen broncodilatación mientras que la budesonida reduce la inflamación.

La indicación autorizada es el tratamiento de mantenimiento en pacientes adultos con enfermedad pulmonar obstructiva crónica de moderada a grave, que no han respondido adecuadamente con a) una combinación de corticoides inhalados y agonistas beta2 adrenérgicos de larga duración; o con b) una combinación de agonistas beta2 adrenérgicos de larga duración y antagonistas muscarínicos de larga duración.

La autorización se ha basado en los resultados de eficacia y seguridad de dos ensayos clínicos aleatorizados, doble ciego, comparados con placebo (ETHOS y KRONOS), que mostraron eficacia tanto en exacerbaciones como en distintas mediciones de la función pulmonar. 
Los efectos adversos más frecuentes fueron neumonía (4,6\%), cefalea $(2,7 \%)$ e infecciones del tracto urinario (2,7\%). En la bibliografía se anexa el plan de gestión de riesgo de este medicamento tras su comercialización establecido por la EMA.

La forma farmacéutica de presentación es la suspensión para inhalación en envase a presión.

\section{INCLISIRÁN $\left(\text { Leqvio }^{\circledR}\right)^{1,24-26}$}

Inclisirán es un agente modificador de lípidos, reduce la acción de la enzima PCSK9 intrahepática y aumenta el reciclado y expresión de los receptores de LDL en la superficie del hepatocito, potenciando así la captación de colesterol-LDL y reduciendo su nivel en sangre.

La indicación autorizada es como complemento a la dieta en adultos con hipercolesterolemia primaria (sea o no del tipo familiar heterocigoto) o dislipidemia mixta; prescribiéndose de dos formas: a) en combinación con una estatina o con una estatina y otra medicación hipolipemiante, en pacientes que no consigan alcanzar los objetivos de colesterol LDL con la dosis máxima tolerada de una estatina; b) Solo o en combinación con otras terapias hipolipemiantes, en pacientes con intolerancia a las estatinas o en los que el uso de estatinas esté contraindicado.

En los ensayos clínicos, aleatorizados, dobles ciegos, comparados con placebo (ORION-10 y ORION-11), ha mostrado que reduce los niveles de LDL-c, en aproximadamente un 50\% de los niveles basales al comparar tratamiento experimental con placebo, y administrándolo de forma subcutánea el día 1, día 90 y posteriormente día 270 y día 450 (es decir, cada 6 meses).

Los efectos adversos más frecuentes fueron reacciones locales (en la zona de inyección), en general leves o moderadas, transitorias, que se resolvieron sin dejar secuelas. En la bibliografía se anexa el plan de gestión de riesgo de este medicamento tras su comercialización establecido por la EMA.

La forma farmacéutica de presentación es como solución inyectable.

\section{LUMASIRAN $\left(\text { Oxlumo }{ }^{\circledR}\right)^{1,27-29}$}

El principio activo es lumasiran, un pequeño ARN de interferencia que degrada el ARN mensajero implicado en la síntesis de la enzima glicolato oxidasa en el hígado, llevando a una reducción de esta enzima. Como resultado se produce una reducción de la concentración de oxalato en plasma y orina, causa subyacente de las manifestaciones de la hiperoxaluria tipo 1 .

La indicación autorizada es el tratamiento de hiperoxaluria tipo 1 en todos los grupos de edad.

La aprobación se ha basado en los resultados de eficacia y seguridad de los estudios ILLUMINATE-A e ILLUMINATE-B. En ILLUMINATE-A, el 84\% de los adultos y niños de $\geq$ seis años que fueron tratados con lumasiran alcanzaron niveles normales o casi normales de oxalato urinario y un 52\% alcanzaron la normalización, en comparación con el $0 \%$ en el grupo placebo. En el estudio ILLUMINATE-B, los resultados de eficacia y el perfil de seguridad de lumasiran en bebés y niños menores de seis años fueron similares a los observados en ILLUMINATE-A.
Lumasiran recibió la designación de medicamento prioritario, y medicamento huérfano en la Unión Europea, y se autorizó a través de una «evaluación acelerada» por parte de la EMA.

Los efectos adversos más frecuentes fueron las reacciones locales en la zona de inyección y dolor abdominal. En la bibliografía se anexa el plan de gestión de riesgo de este medicamento establecido por la EMA, tras su comercialización.

La forma farmacéutica de presentación es como solución inyectable.

\section{POLVO DESENGRASADO DE ARACHIS HYPOGAEA L. (SEMILLA DE CACAHUETE) ( Palforzia $\left.^{\circledR}\right)^{1,30-32}$}

El principio activo es polvo desengrasado de Arachis hypogaea L., semilla de cacahuete, un alérgeno que se administra en dosis crecientes a pacientes con alergia al cacahuete con el fin de que el sistema inmune aprenda a tolerar pequeñas cantidades de cacahuetes.

La indicación autorizada es alergia confirmada al cacahuete en pacientes de 4 a 17 años, aunque puede seguir prescribiéndose y administrándose a pacientes $\geq$ de 18 años. Este medicamento debe ser utilizado en combinación con una dieta libre de cacahuete y bajo la supervisión del profesional sanitario cualificado en el diagnóstico y tratamiento de enfermedades alérgicas.

Se realizó un estudio multicéntrico, doble ciego aleatorizado frente a placebo $(3: 1)$, como ensayo pivotal para la autorización que valoró eficacia, seguridad y calidad de vida de los pacientes, obteniendo en el grupo experimental que un 58\% (77/132) toleraban $1000 \mathrm{mg}$ de proteína de cacahuete versus el 2\% (1/43) del grupo control (diferencia de medias 56\% IC 95\%: 44,1-65,2).

Los efectos adversos más frecuentes fueron dolor abdominal, irritación de garganta, náuseas, vómitos, urticaria, prurito oral y molestias en la zona superior del abdomen. Se adjunta en referencias bibliográficas el enlace al plan de gestión de riesgo de este medicamento tras su comercialización por parte de la EMA.

Las formas farmacéuticas de presentación son sobres y cápsulas, de administración oral, tras su mezcla con alimentos blandos adecuados a la edad del paciente.

\section{RILPIVIRINA (Rekambys $\left.{ }^{\circledR}\right)^{1,3-6,33}$}

El principio activo es rilpivirina, un antiviral de acción directa Inhibidor de la transcriptasa inversa. La rilpivirina activa la inhibición no competitiva de la transcriptasa inversa del VIH1. Siempre debe ser utilizado en combinación con cabotegravir inyectable, otro antiretroviral de acción prolongada.

Al inicio de esta nota técnica se ha mencionado la autorización para cabotegravir $\left(\right.$ Vocabria $^{\circledR}$ ), otro antirretroviral de acción prolongada, para el que se autorizado al mismo tiempo la combinación estudiada con Rilpavirina, compartiendo la misma indicación como es lógico. La indicación aprobada es, «en combinación con cabotegravir, para el tratamiento de la infección por el virus de inmunodeficiencia humano tipo 1 (VIH-1) en adultos con carga viral indetectable (HIV-1 RNA $<50$ copias $/ \mathrm{mL}$ ) que siguen un régimen antirretroviral estable sin evidencia, ya sea previa o actual, de resistencia o fracaso virológico con inhibidores de 


\section{Evaluación positiva de medicamentos: octubre 2020}

la transcriptasa inversa no análogos de los nucleósidos (ITINN) e inhibidores de la integrasa (INI)».

El uso de esta combinación, como ya se explicó anteriormente permite reducir la frecuencia de administración a una vez al mes o una vez cada dos meses en comparación con la administración oral diaria. Rekambys ${ }^{\circledR}$ estará disponible como suspensión inyectable de liberación prolongada (600 y $900 \mathrm{mg}$ ) y ser prescrito por médicos con experiencia en el manejo de la infección por VIH.

Los datos de eficacia y seguridad son los ya descritos en el apartado dedicado a cabotegravir $\left(\right.$ Vocabria $\left.^{\circledR}\right)$ al inicio de esta revisión.

\section{BIBLIOGRAFÍA}

1. Agencia Española de Medicamentos y Productos Sanitarios (AEMPS). Boletín mensual de la AEMPS sobre medicamentos de uso humano del mes de octubre de 2020. Disponible en: https://www.aemps.gob.es/informa/boletines-aemps/boletinMensual/2020-3/boletin-mensual-de-la-aemps-sobremedicamentos-de-uso-humano-del-mes-de-octubre-de-2020/\#nuevosMed.

2. European Medicines Agency. EPAR-Product Information of Vocabria ${ }^{\circledR}$ Consultado el 25/02/2021.Disponible en: https://www.ema.europa.eu/en/documents/assessment-report/vocabria-epar-public-assessment-report_en.pdf.

3. Orkin C, Arasteh K, Górgolas Hernández-Mora M, Pokrovsky V, Overton ET, Girard PM, et al. Long-Acting Cabotegravir and Rilpivirine after Oral Induction for HIV-1 Infection. N Engl J Med. 2020 Mar 19; 382(12): 1124-1135. doi: 10.1056/NEJMoa1909512. Epub 2020 Mar 4. PMID 32130806 .

4. Swindells S, Andrade-Villanueva JF, Richmond GJ, Rizzardini G, Baumgarten A, Masiá M, et al. Long-Acting Cabotegravir and Rilpivirine for Maintenance of HIV-1 Suppression. N Engl J Med. 2020 Mar 19; 382(12): 1112 1123. doi: 10.1056/NEJMoa1904398. Epub 2020 Mar 4. PMID: 32130809

5. Margolis DA, Gonzalez-Garcia J, Stellbrink HJ, Eron JJ, Yazdanpanah Y, Podzamczer D, et al. Long-acting intramuscular cabotegravir and rilpivirine in adults with HIV-1 infection (LATTE-2): 96-week results of a randomised, open-label, phase 2b, non-inferiority trial. Lancet. 2017 Sep 23; 390(10101): 1499-1510. doi: 10.1016/S0140-6736(17)31917-7. Epub 2017 Jul 24. PMID 28750935.

6. Markowitz M, Frank I, Grant RM, Mayer KH, Elion R, Goldstein D, et al. Safety and tolerability of long-acting cabotegravir injections in HIV-uninfected men (ECLAIR): a multicentre, double-blind, randomised, placebo-controlled, phase 2a trial. Lancet HIV. 2017 Aug; 4(8): e331-e340. doi: 10.1016/ S2352-3018(17)30068-1. Epub 2017 May 22. PMID: 28546090.

7. European Medicines Agency. EPAR-Product Information of Tecartus ${ }^{\circledR}$. Consultado el 28/02/2021.Disponible en: https://www.ema.europa.eu/en/documents/assessment-report/tecartus-epar-public-assessment-report_en.pdf.

8. European Medicines Agency. Orphan Maintenance Assessment Report- Tecartus $^{\circledR}$. Consultado el 25/02/2021.Disponible en:https://www.ema.europa. eu/en/documents/orphan-maintenance-report/tecartus-orphan-maintenance-assessment-report_en.pdf.

9. Wang M, Munoz J, Goy A, Locke FL, Jacobson CA, Hill BT, et al. KTE-X19 CAR T-Cell Therapy in Relapsed or Refractory Mantle-Cell Lymphoma. N Engl J Med. 2020 Apr 2; 382(14): 1331-1342. doi: 10.1056/NEJMoa1914347. PMID: 32242358; PMCID: PMC7731441.

10. European Medicines Agency. EPAR-Product Information of Libmeldy ${ }^{\circledR}$ Consultado el 01/03/2021.Disponible en: https://www.ema.europa.eu/en/documents/product-information/libmeldy-epar-product-information_en.pdf.

11. European Medicines Agency. Orphan Maintenance Assessment Report- Libmeldy ${ }^{\mathbb{R}}$. Consultado el 01/03/2021.Disponible en:https://www.ema.europa. eu/en/documents/orphan-maintenance-report/libmeldy-orphan-maintenance-assessment-report_en.pdf.

12. Biffi A, Montini E, Lorioli L, Cesani M, Fumagalli F, Plati T, et al. Lentiviral hematopoietic stem cell gene therapy benefits metachromatic leukodystrophy. Science. 2013 Aug 23; 341(6148): 1233158. doi: 10.1126/science.1233158. Epub 2013 Jul 11. PMID: 23845948.
13. Sessa M, Lorioli L, Fumagalli F, Acquati S, Redaelli D, Baldoli C, et al Lentiviral haemopoietic stem-cell gene therapy in early-onset metachromatic leukodystrophy: an ad-hoc analysis of a non-randomised, open-label, phase 1/2 trial. Lancet. 2016 Jul 30; 388(10043): 476-87. doi: 10.1016/S01406736(16)30374-9. Epub 2016 Jun 8. PMID: 27289174.

14. European Medicines Agency. EPAR-Public Assessment Report - Fintepla ${ }^{\circledR}$. Consultado el 05/03/2021.Disponible en: https://www.ema.europa.eu/en/documents/assessment-report/fintepla-epar-public-assessment-report_en.pdf.

15. European Medicines Agency. Orphan Maintenance Assessment Report- Fintepla $^{\circledR}$. Consultado el 05/03/2021. Disponible en: https://www.ema.europa. eu/en/documents/assessment-report/fintepla-epar-public-assessment-report en.pdf.

16. Lagae L, Sullivan J, Knupp K, Laux L, Polster T, Nikanorova M, et al. Fenfluramine hydrochloride for the treatment of seizures in Dravet syndrome: a randomised, double-blind, placebo-controlled trial. Lancet. 2019 Dec 21; 394(10216): 2243-2254. doi: 10.1016/S0140-6736(19)32500-0. Epub 2019 Dec 17. PMID: 31862249.

17. Pierce JG, Mithal DS. Fenfluramine: New Treatment for Seizures in Dravet Syndrome. Pediatr Neurol Briefs. 2020 Mar 12; 34: 8. doi: 10.15844/pedneurbriefs-34-8. PMID: 32189958; PMCID: PMC7071945.

18. Schoonjans A, Paelinck BP, Marchau F, Gunning B, Gammaitoni A, et al. Low-dose fenfluramine significantly reduces seizure frequency in Dravet syndrome: a prospective study of a new cohort of patients. Eur J Neurol 2017 Feb; 24(2): 309-314. doi: 10.1111/ene.13195. Epub 2016 Oct 28. PMID: 27790834; PMCID: PMC5298030.

19. Sharawat IK, Panda PK, Kasinathan A, Panda P, Dawman L, Joshi K. Efficacy and tolerability of fenfluramine in patients with Dravet syndrome: A systematic review and meta-analysis. Seizure. 2021 Feb; 85: 119-126. doi: 10.1016/j.seizure.2020.12.016. Epub 2021 Jan 11. PMID: 33461030.

20. European Medicines Agency. EPAR-Public assesment report of Trixeo Aerosphere $^{\circledR}$. Consultado el 06/03/2021. Disponible en: https://www.ema. europa.eu/en/documents/assessment-report/trixeo-aerosphere-epar-publicassessment-report_en.pdf.

21. European Medicines Agency. EPAR-Risk-Management-Plan Summary of Trixeo Aerosphere ${ }^{\circledR}$. Consultado el 06/03/2021. Disponible en: https://www. ema.europa.eu/en/documents/rmp-summary/trixeo-aerosphere-epar-riskmanagement-plan-summary_en.pdf.

22. Rabe KF, Martinez FJ, Ferguson GT, Wang C, Singh D, Wedzicha JA, et al. A phase III study of triple therapy with budesonide/glycopyrrolate/ formoterol fumarate metered dose inhaler $320 / 18 / 9.6 \mu \mathrm{g}$ and $160 / 18 / 9.6 \mu \mathrm{g}$ using co-suspension delivery technology in moderate-to-very severe COPD: The ETHOS study protocol Respir Med. 2019 Oct-Nov; 158: 59-66. doi: 10.1016/j.rmed.2019.08.010. Epub 2019 Aug 22. PMID: 31605923.

23. Ferguson GT, Rabe KF, Martinez FJ, Fabbri LM, Wang C, Ichinose M, et al. Triple Therapy with budesonide/glycopyrrolate/formoterol fumarate with co-suspension delivery technology versus dual therapies in chronic obstructive pulmonary disease (KRONOS): a double-blind, parallel-group, multicentre, phase 3 randomised controlled trial. Lancet Respir Med. 2018 Oct;6(10):747-758. doi:10.1016/S2213-2600(18)30327-8. Epub 2018 Sep 16 Erratum in: Lancet Respir Med. 2018 Oct 4; Erratum in: Lancet Respir Med. 2019 Feb;7(2):e9. PMID: 30232048.

24. European Medicines Agency. EPAR- Public assesment report of Leqvio ${ }^{\circledR}$. Consultado el 03/03/2021. Disponible en: https://www.ema.europa.eu/en/ documents/assessment-report/leqvio-epar-public-assessment-report_en.pdf.

25. European Medicines Agency. EPAR-Risk-Management-Plan Summary of Leqvio $^{\circledR}$. Consultado el 03/03/2021. Disponible en: https://www.ema.europa. eu/en/documents/rmp-summary/leqvio-epar-risk-management-plan-summary_en.pdf.

26. Ray KK, Wright RS, Kallend D, Koenig W, Leiter LA, Raal FJ, et al. Two Phase 3 Trials of Inclisiran in Patients with Elevated LDL Cholesterol. N Engl J Med. 2020 Apr 16; 382(16): 1507-1519. doi:10.1056/NEJMoa1912387. Epub 2020 Mar 18. PMID: 32187462.

27. European Medicines Agency. EPAR-Public assesment report of Oxlumo ${ }^{\circledR}$. Consultado el 03/03/2021Disponible en: https://www.ema.europa.eu/en/documents/assessment-report/oxlumo-epar-public-assessment-report_en.pdf.

28. European Medicines Agency. EPAR-Risk-Management-Plan Summary of Oxlumo $^{\circledR}$. Consultado el 03/03/2021.Disponible en: https://www.ema.europa. eu/en/documents/rmp-summary/oxlumo-epar-risk-management-plan-summary_en.pdf. 
Aparicio-Hernández R., et al.

29. Scott LJ, Keam SJ. Lumasiran: First Approval. Drugs. 2021 Feb; 81(2): $277-$ 282. doi: 10.1007/s40265-020-01463-0. PMID: 33405070.

30. European Medicines Agency. EPAR-Public assesment report of Palforzia ${ }^{\circledR}$. Consultado el 02/03/2021. Disponible en: https://www.ema.europa.eu/en/documents/assessment-report/palforzia-epar-public-assessment-report_en.pdf.

31. European Medicines Agency. EPAR-Risk-Management-Plan Summary of Palforzia ${ }^{\circledR}$ Consultado el 02/03/2021. Disponible en: https://www.ema.europa.eu/en/documents/rmp-summary/palforzia-epar-risk-management-plansummary_en.pdf.
32. O’B Hourihane J, Beyer K, Abbas A, Fernández-Rivas M, Turner PJ, Blumchen K, et al. Efficacy and safety of oral immunotherapy with AR101 in European children with a peanut allergy (ARTEMIS): a multicentre, doubleblind, randomised, placebo-controlled phase 3 trial. Lancet Child Adolesc Health. 2020 Oct; 4(10): 728-739. doi: 10.1016/S2352-4642(20)30234-0. Epub 2020 Jul 20. PMID: 32702315.

33. European Medicines Agency. EPAR-Public assesment report of Rekambys ${ }^{\circledR}$. Consultado el 06/03/2021. Disponible en: https://www.ema.europa.eu/en/documents/assessment-report/rekambys-epar-public-assessment-report_en.pdf. 